\title{
An Effective Classification of Citrus Fruits Diseases using Adaptive Gamma Correction with Deep Learning Model
}

\author{
C. Senthilkumar, M. Kamarasan
}

\begin{abstract}
In farming sector, diseases affected in plants are mainly accountable for the minimized profit that leads to financial loss. In case of plants, citrus is utilized as a main resource of nutrients namely vitamin $C$ globally. But citrus diseases greatly affect the productivity as well as quality. In recent days, computer vision and image processing approaches are commonly applied for detecting and classifying the plant diseases. This paper presents a novel deep learning (DL) based citrus disease detection and classification model. A new DL based AlexNet architecture is employed for effective identification of diseases. The presented model involves four main processes namely pre-processing, segmentation, feature extraction, and classification. Initially, pre-processing takes place to improve the quality of the image. Then, the Otsu method is applied to segment the images. Next, Alex-Net model is applied as a feature extractor. Finally, random forest (RF) classifier is used to classify the different kinds of citrus diseases. Besides, adaptive gamma correction (AGC) model is applied to improve the contrast of the applied citrus images. A comprehensive experimentation takes place on Citrus Disease Image Gallery Dataset. The results are examined under several cases and the outcome ensured the effective characteristics of the presented AGC-A model.
\end{abstract}

Keywords: Alex Net, Citrus disease, Deep learning, Gamma correction.

\section{INTRODUCTION}

Plants which yield fruits are the main part of agro-economical society. Among several other fruit plants, citrus fruits as well as plants are composed with Vitamin-C, that offers several advantages for human health and used as source material in many agro industries. Moreover, production of citrus are affected globally due to the citrus disease. Some of the citrus diseases are greasy spot, black spot and canker. Presently, many other automated decision-making models are presented for detecting symptoms of citrus disease, and challenging outcome are added. Recently, many researchers trying to

Revised Manuscript Received on December 30, 2019.

* Correspondence Author

C. Senthilkumar*, Research Scholar, Department of Computer and Information Science, Annamalai University, Chidambaram (Tamil Nadu) India. E-mail:senthilkumar_c@hotmail.com

M. Kamarasan, Assistant professor, Department of Computer and Information Science, Annamalai University, Chidambaram (Tamil Nadu) India. E-mail: smkrasan@yahoo.com

(C) The Authors. Published by Blue Eyes Intelligence Engineering and Sciences Publication (BEIESP). This is an open access article under the CC BY-NC-ND license (http://creativecommons.org/licenses/by-nc-nd/4.0/) identify new machine based solution in order to predict the citrus disease primarily, from the field image processing. Since the citrus lesion spot detecting and classifying process comprises of Pre-processing, Segmentation, Feature extraction and classification. For detecting infected area on plant leaves, diverse classification models are utilized in the field of image processing. Alike, to spot segmentation of disease and classifying citrus fruits and leaves, diverse methods are executed. For lesion spot segmentation, K-means clustering, region-based segmentation, Otsu thresholding, edge-based and region-oriented segmentation methods are used. For classifying process, massive feature extraction as well as supervised machine learning approaches is utilized. Therefore, different methods incorporated, K-nearest Neighbours (KNN), Linear Discriminant Analysis (LDA), Naive Bayes (NBC), Back Propagation Neural Networks (BPNN) and Random Forests (RFT) have been exploited. The above study is addressed with different limitations. For example, contrast expansion is required when contrast lesion spot is lower, since it influences the accuracy of lesion segmentation and decrease classification accuracy. Hence, effective feature extracting, and selection techniques are important for those application because each disease has unique spots and symptoms. This literature has several issues, some of them are: local noise because of capturing gadgets and poor atmosphere, orientation modification, color and texture of symptoms. By considering the above-mentioned facts, citrus fruits are an important fruit in agricultural economy which requires some models for minimizing the failure caused by citrus disease in plants. From the domain of image processing and machine learning (ML) techniques, various methods for detecting and classifying citrus disease are presented. [1] introduced an automatic system to detect, segment and measure citrus fruits. This technique comprises of preprocessing, shadow reduction, separating the object, K-means clustering, and blob detection stages. Regarding this, [2] requested a method from computer vision. Hence, it enhances the opposing effect established through the shape of spherical object at image acquisition phase. [3] proposed an approach for measuring the mass and size of 4 different citrus fruits namely oranges, limes, lemons and tangerines. It helps to estimate the accumulation and size of the fruits. [4] deploys a scheme to sort and colour-based grading of infected citrus fruit. By using the proposed method, citrus fruits that are combined with other fruits are identified efficiently. 
This technique classifies fruits into various groups on the basis of Gray-Level Co-Occurrence Matrix (GLCM) variable. [5] introduces a new approach for detecting and classifying citrus diseases with the help of $\Delta \mathrm{E}$ colour difference technique for detecting the affected parts in the image. It uses HOG, colour and texture features for classifying the citrus diseases. The simulation outcome represented that the proposed method considerably enhances the operation in terms of accuracy and AUC. [6] designed a novel technique for predicting citrus canker. This technique exploits the combination of fluorescence imaging spectroscopy and ML techniques like SVM for detecting canker. It detects the citrus diseases efficiently, such as scab and canker, also differentiates among them. [7] developed a new scheme for citrus greening detection on the basis of huanglongbing (HLB) and SVM. The citrus leaves are gathered below the lighting condition for extracting features of colour, texture, and HOG. PCA is used for reducing the extracted features and rate SVM for classification. Based on the outcome, rate SVM classifying unit accomplished 91.93\% accuracy under low cost and minimum processing time when compared with other classification models. [8] projected a method for automatic detection and classification of citrus diseases. The above technique consists of 2 stages. At the first stage, feature selection technique is obtained that again contains two primary steps including feature ranking and threshold based optimal feature selection. At the second stage, the chosen features undergo classification through several classifier models like Naive Bayes, CART and NN with the help of multiple perceptron learning and attained around $88 \%$ of accuracy for every predicted disease.

[9] employs a new model for auto detection of citrus canker from leaf images with the combination of global and local features. The proposed model utilizes an enhanced Ada-boost method for selecting the prominent features. Consequently, colour and local texture features are filtered and classified using NN, SVM, and KNN. [10] establishes some other models. This method exploits leaves based feature inspection for primary detection of citrus diseases. It operates in four different phases. At the first phase, input image is hold with the help of digital camera and pre-process with enhancing and colour space structure is performed. Second phase, segmentation of the defected area by K-means clustering is performed. Third phase, extracting the GLCM features. Last phase performs SVM-based classification for discovering the affected leaf image. Commonly used segmentation methods are, thresholding, K-means clustering, region growing, and morphological based models has massive number of limitations. For example, these techniques do not perform well whenever the input image is too much difficult, and contain poor contrast. GLCM features do not help high classification rate when compared with colour features, due to diverse colour of disease spots. Therefore, many other problems that decrease the accuracy of system. The issues are poor contrast problem, extraction of efficient features, selection of most prominent features and irregularity in disease part.

In recent days, computer vision and image processing approaches are commonly applied for detecting and classifying the plant diseases. This paper presents a novel deep learning (DL) based citrus disease detection and classification model. A new DL based AlexNet architecture is employed for effective identification of diseases. Besides, adaptive gamma correction (AGC) model is applied to improve the contrast of the applied citrus images. A comprehensive experimentation takes place on Citrus Disease Image Gallery Dataset. The results are examined under several cases and the outcome ensured the effective characteristics of the presented AGC-A model.

\section{THE PROPOSED AGC-A MODEL}

The working process of the presented AGC-A model is demonstrated in Fig. 1. As shown, it involves four main processes namely pre-processing, segmentation, feature extraction, and classification. Initially, pre-processing takes place to improve the quality of the image. Then, the Otsu method is applied to segment the images. Next, Alex-Net model is applied as a feature extractor. Finally, RF classifier is used to classify the different kinds of citrus diseases.

\section{A. Preprocessing}

Pre-processing works on two main levels namely contrast enhancement (CE) and noise removal. Initially, the CE takes place using AGC model. Next, the noises present in the image are eliminated by the use of bilateral filtering (BF). Then, the smoothing of the images takes place.

\section{1) AGC Based CE}

AGC technique is presented with connecting the gamma parameter by cumulative distributive function (CDF) [11]. The changed pixel intensity $\mathrm{T}(i)$ are calculated as

$$
\begin{aligned}
& \mathrm{T}(i)=\text { round }\left[i_{\mathrm{mx}}\left(\frac{i}{i_{\mathrm{mx}}}\right)^{y(i)}\right] \\
& y(i)=1-c(i)=1-\sum_{x=0}^{i} p(x)
\end{aligned}
$$

Where $i=0,1, \ldots, 255$ the CDF of gray levels are in the applied image, $p(x)$ indicates the stabilized gray level histogram. round $[\cdot]$ indicates the rounding process. Now, 8-bit grayscale image under highest pixel intensity $i_{\mathrm{mx}}=$ 255 is considered. As exposed in Fig. 2, the gamma value $y(i)$ monotonically drops from 1 to 0 . By itself, the least value pixels in reduced images would be stretched.

The pixel dynamic range of resultant images will be covered which CE can be attained. Still, a weighting distribution method is used for smoothing the primary histogram as defined as

$$
p_{w}(i)=p_{\mathrm{mx}}\left(\frac{p(i)-p_{m n}}{p_{\mathrm{mx}}-p_{\mathrm{mn}}}\right)^{\alpha}
$$

where $\alpha$ is the modifiable parameter, $p_{\mathrm{mx}}=\operatorname{mx} p(i)$, $p_{\mathrm{mn}}=\operatorname{mn} p(i)$. Then $p_{w}(i)$ are standardized yielding $p_{w}^{\prime}(i)$. The histogram weighted with $\alpha<1$ develops relatively plane and smooth at less intensities, so as to the adverse outcome is declined. 


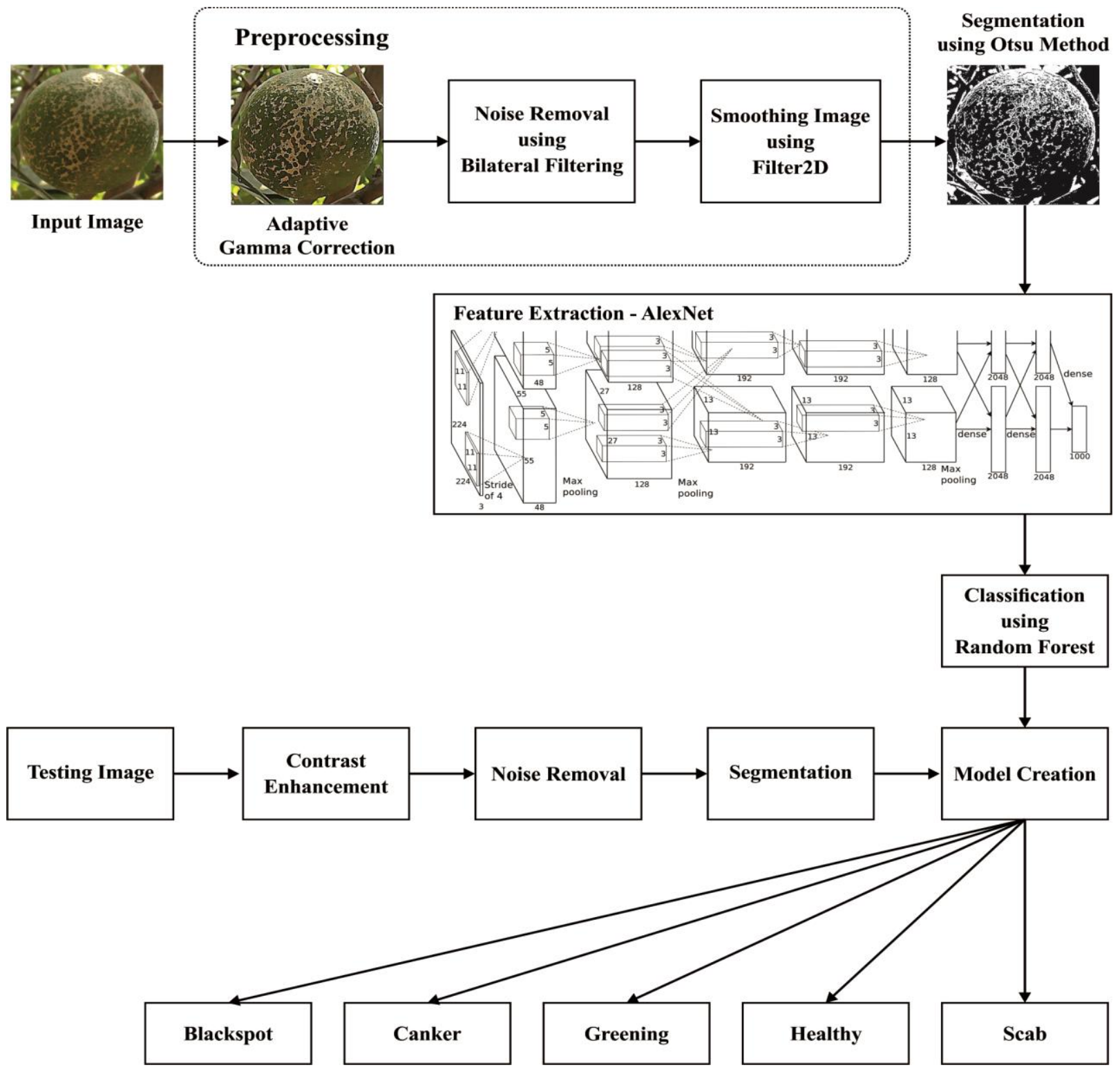

Fig.1. Working principle of AGC-A method

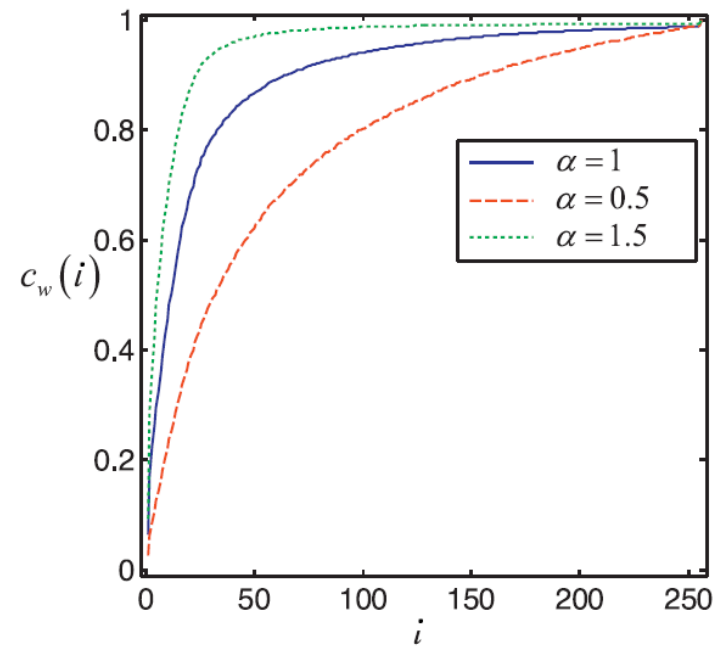

Fig.2. AGC based CE with varying levels of $\alpha$

\section{2) Bf Based Noise Removal}

Here, $\mathrm{BF}$ is applied on the test images which are in gray scale [12]. Generally, some amount of noise exists in the image leads to misidentification of the plant diseases. The likelihood of BF is based on a particular weighting element for averaging nearby pixels to remove the noise.The general representation of BF includes a distance-based domain filter part $\left(i, i^{\prime}\right)$, and a gray-value dependent range filter part $r\left((f i),\left(f i^{\prime}\right)\right)$ :

$$
\begin{gathered}
\tilde{f}(i)=\frac{1}{N(x)} \int_{-\infty}^{\infty} f\left(i^{\prime}\right) d\left(i, i^{\prime}\right) r\left(f(i), f\left(i^{\prime}\right)\right) d i^{\prime} \\
d\left(i, i^{\prime}\right) \propto \exp \left(-\frac{\left(i-i^{\prime}\right)^{2}}{2 \sigma_{d}^{2}}\right) \\
r\left(f(x), f\left(x^{\prime}\right)\right) \propto \exp \left(-\frac{\left(f(x)-f\left(x^{\prime}\right)\right)^{2}}{2 \sigma_{f}^{2}}\right)(6)
\end{gathered}
$$

with $\sigma_{d}$ is a width parameter of the filter kernel size and $\sigma_{f}$ the noise standard deviation of the considered reconstruction value. 


\section{B. Otsu Based Segmentation Model}

Otsu's method which is organized using many kinds of image processing applications for executing histogram based image thresholding or converts a gray level image to binary image [13]. This technique assumes that image embraces bi-modal histogram and estimates a best threshold, portioning aforementioned 2 classes, to be negligible regarding their joint spread or intra-class variance. Extending the fundamental scheme to multi-level thresholding is said to be Multi Otsu method. Otsu's method explores the threshold at minimum inter-class variance very minutely, described as weighted sum of variances of two classes:

$$
\sigma_{\omega}^{\mathrm{b}}(\mathrm{t})=\omega_{0}(\mathrm{t}) \sigma_{0}^{\mathrm{b}}(\mathrm{t})+\omega_{\mathrm{a}}(\mathrm{t}) \sigma_{\mathrm{a}}^{\mathrm{b}}(\mathrm{t})
$$

Aforementioned weights $\omega_{0}$ and $\omega_{\mathrm{a}}$ are possibilities of two classes independent by a threshold $t$ and $\sigma_{0}^{\mathrm{b}}$ and $\sigma_{\mathrm{a}}^{\mathrm{b}}$ are variances of these two classes.Otsu has a template, which reduces the intra-class variance that is correlated to maximum inter-class variance as:

$$
\sigma_{\mathrm{y}}^{\mathrm{b}}(\mathrm{t})=\sigma^{\mathrm{b}}-\sigma_{\omega}^{\mathrm{b}}(\mathrm{t})=\omega_{\mathrm{a}}(\mathrm{t}) \omega_{\mathrm{b}}(\mathrm{t})\left[\mu_{\mathrm{a}}(\mathrm{t})-\mu_{\mathrm{b}}(\mathrm{t})\right](8)
$$

They are denoted in terms of class possibilities $\omega_{\mathrm{a}}$, and class means $\mu_{\mathrm{a}}$.

The class probability $\omega_{\mathrm{a}}(\mathrm{t})$ will be estimated from histogram $t$ as:

$$
\omega_{\mathrm{a}}(\mathrm{t})=\sum_{0}^{t} \mathrm{P}(\mathrm{i})
$$

Whereas, the class mean $\mu_{\mathrm{l}}(\mathrm{t})$ is:

$$
\mu_{\mathrm{a}}(\mathrm{t})=\sum_{0}^{t} \mathrm{P}(\mathrm{i}) \mathrm{x}(\mathrm{i})
$$

Where $\mathrm{x}(\mathrm{i})$ is the value in the middle of histogram bin.Class probabilities and class means are processed frequently that produces effective technique.

\section{AlexNet based Feature Extractor model}

The AlexNet architectures have eight learned layers-five convolutional and three fully connected (FC) layers [14].

\section{ReLU Nonlinearity}

The standard way to represent a neuron's output $f$ as a function of its input $i$ is with $f(i)=\tanh (i)$ or $f(i)=(1+$ $\left.e^{-i}\right)^{-1}$. In conditions of trained timing through gradient descent, this saturate nonlinearity is much lesser than the non-saturate nonlinearity $(i)=\mathrm{mx}(0, i)$. Subsequent, the neurons with this nonlinearity are referred as ReLU. Deep CNNs through ReLUs training in various times are quicker than their corresponding tanh units. The nonlinearity $f(i)=$ | tanh $(i) \mid$ works mostly well by their type of contrast normalization followed with local average pooling. Still, the primary concerns are avoiding over fitting, thus the outcome is examined to accelerate capability to fit the training set. Quicker learning has a great influence on the action of large concepts trained on huge databases.

\section{Training on Multiple GPUs}

The single GTX 580 GPU has only $3 G B$ of memory that limits the highest size of the networks that can be trained. It rotates out that 1.2 million trained examples which is enough to train networks that is too high to fit on one GPU.
Consequently, we extend the net crosswise 2 GPUs. The parallelization system which can utilize effectively sets half of the kernels (or neurons) on every GPU by one extra trick: the GPUs correspond only in definite layers. For example, in layer 2 all kernel maps are given input of layer 3. Still, the layer 3 kernel maps are given input only to the layer 4 , that exist in the same GPU. Selecting the design of connectivity is the drawback to cross validation, however this lets us to accurately tune the quantity of transmission till it is a suitable fraction of the quantity of computation.The ensuing designs are rather like to which of the "columnar" CNN utilized, but the column is not independent. This method decreases thetop-5 and top-1 fault rate with $1.2 \%$ and $1.7 \%$, correspondingly, as evaluated through a net by half as several kernels in every CONV layer to train on one GPU. The 2 GPU net gets somewhat small duration to train than the 1 GPU net.

\section{Local Response Normalization}

ReLUs has the attractive assets which they don't need input normalization to avoid them from oversupplying. In any case, some training examples make a positive input to a ReLU, learning will occur in which neuron. Still, we get that the pursuing local normalization system aids generalization. Indicating with $a_{i, j}^{x}$ the action of a neuron employed with concerning kernel $x$ at position $(i, j)$ with the concerning the ReLU non linearity, the reply normalized action $b_{i, j}^{x}$ are provided with the expression

$$
b_{i, j}^{x}=a_{i, j}^{x} /\left(k+\alpha \sum_{y=\operatorname{mx}\left(0, x-\frac{n}{2}\right)}^{\operatorname{mn}\left(N-1, x+\frac{n}{2}\right)}\left(a_{i, j}^{y}\right)^{2}\right)^{\beta}
$$

where the sum runs above $n$ "adjacent" kernel maps by the similar spatial stage, and $N$ are the entire number of kernels in these layers. To arranging the kernel maps are certainly random and decided to train before. This arrangement of reply normalization executes a variety of lateral inhibition encouraged with the type create in actual neurons; generating contest for large actions between neuron results calculated with various kernels. The constants $k, n, \alpha$, and $\beta$ is hyper parameters those value is verified by a validation set; we use $k=2, n=5, \alpha=10^{-4}$, and $\beta=0.75$. To apply this normalization following concern the ReLU non-linearity in definite layers. This system allows several similarities to the local contrast normalization method, however we will be further properly termed "brightness normalization", as we don't take away the mean action. Reply normalization decreases the top-5 and top- 1 fault rates with $1.2 \%$ and $1.4 \%$, correspondingly.

\section{Overlapping Pooling}

Pooling layers in CNNs review the results of nearby sets of neurons in the similar kernel map. Usually, the regions reviewed with nearby pooling units don't overlap. To be further accurate, a pooling layer can be included consists of a grid of pooling units spaced $s$ pixels separately, Every region of size $t \times t$ is reviewed in the middle position of a pooling unit. When $s=t$, conventional local pooling is attained as usually utilized in CNNs.

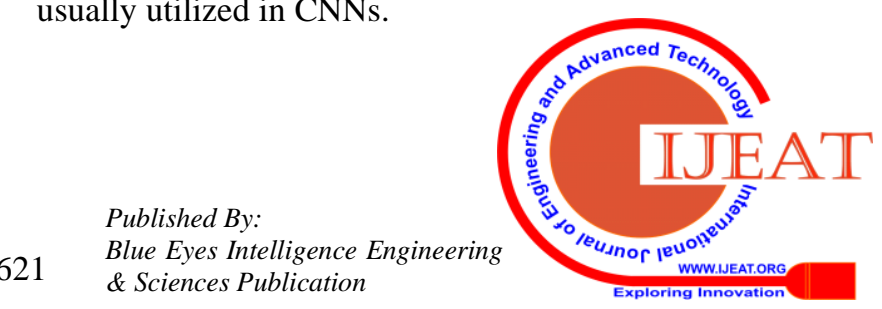


When $s<t$, overlap pooling is attained. There is what AlexNet is used by $s=2$ and $t=3$. This method decreases the top- 1 and top-5 fault rates with $0.4 \%$ and $0.3 \%$, correspondingly, as evaluated through the non overlapping method $s=2, t=2$ that makes the result of corresponding dimensions.

\section{Overall Architecture}

As represented in Fig. 3, the net has 8 layers through weights; the $1^{\text {st }}$ five is CONV and the left behind three is FC. The results of the final FC layers are fed to a 1000way softmax that generates an allocation above the 1000 classes' label. AlexNet exploits the multinomial logistic failure objectives that are corresponding to exploit the average across the trained cases of the logpossibility of the exact label below the prediction allocation. The kernel of the second, fourth, and fifth CONV layer is attached only to individuals kernel maps in the before layer that exist on the similar GPU. The kernel of a third convolution layer is attached to every kernel maps in the $2^{\text {nd }}$ layer. The neuron in the FC layer is attached to every neuron in the before layer. Reply normalization layers pursue the $1^{\text {st }}$ and $2^{\text {nd }}$ CONV layers. Max pooling layers, to the type, follow together reply normalization layers with the $5^{\text {th }}$ CONV layer. The ReLU nonlinearities are concerned to the result of each CONV and FC layer. The $1^{\text {st }} \mathrm{CONV}$ layer exacts the $224 \times 224 \times 3$ input picture through 96 kernels of size $11 \times 11 \times 3$ by a stride of 4 pixels (there is the distance among the accessible field middles of nearby neurons in a kernel map). The 2nd CONV layer takes as input (response-normalized and pooled) output of the first CONV layer. The third, fourth, and fifth CONV layers are attached to one another not including some prevailing pooling or normalization layers. The third CONV layers have 384 kernels of size $3 \times 3 \times 256$ attached to the (normalized, pooled) results of the second CONV layer. The fourth CONV layers have 384 kernels of size $3 \times 3 \times 192$, and the 5 th CONV layer have 256 kernels of size $3 \times 3 \times 192$. The FC layer has 4096 neurons every.

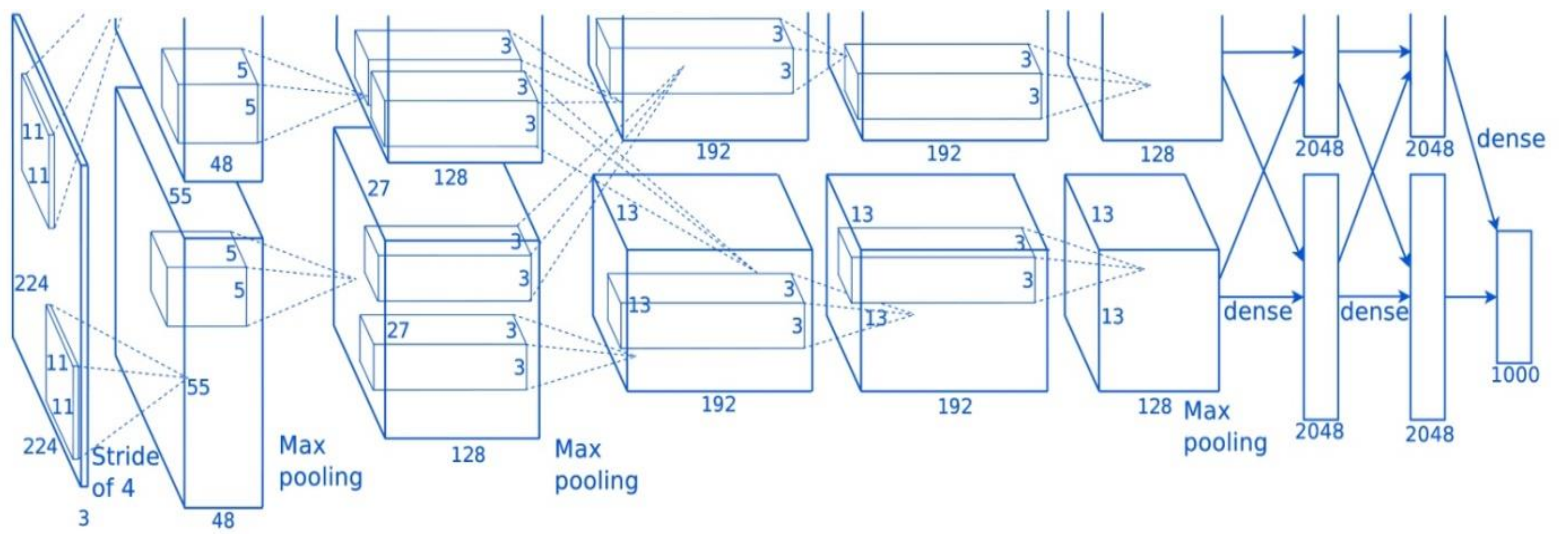

Fig.3. Alex Net Architecture

\section{RF classifier}

In 2001, Breiman presented a novel and significant tree-based ensemble classification model [15] depending upon the integration tree of predictors so that every tree is based on the values of a arbitrary vector which undergo sampling in an independent way and with the identical distribution for every tree and is referred as RF. It comprises an integration of separate base classifiers where every tree is created utilizing a using a random vector sampled in an independent way from the classifier input vector for enabling a quicker generation of tree. To classify the data, the classification individual vote from every tree is integrated by the use of a rule-based model.

\section{EXPERIMENTAL VALIDATION}

\section{A. Dataset Description}

For examining the performance of the applied AGC-A model, it is tested against Citrus Image Gallery dataset. The dataset comprises a total of 150 citrus fruits images. Among them, a set of 19 images comes under the black spot disease category, 78 images fall into the canker disease category, 16 images comes under the greening disease type, 15 and 22 images comes under the category of scab and healthy [16].

Table 1 Dataset details

\begin{tabular}{|c|c|}
\hline Citrus Fruits Disease & Image count \\
\hline Black Spot & 19 \\
\hline Canker & 78 \\
\hline Greening & 16 \\
\hline Scab & 15 \\
\hline Healthy & 22 \\
\hline Total Images & $\mathbf{1 5 0}$ \\
\hline
\end{tabular}

\section{B. Results analysis}

The effectiveness of the presented AGC-A model is validated under several aspects. Table 2 displayed the images obtained from the CE process. The images in Table (a-c) and (g-i) indicates the healthy images. The corresponding CE images are found in the Table (d-f) and (j-l) respectively. The figures clearly indicate that the contrast level of the images are clearly enhanced by the AGC method which will helps to improvise the detection performance. 
Table 2 Results of Contrast Enhancement using Adaptive Gamma Correction Method

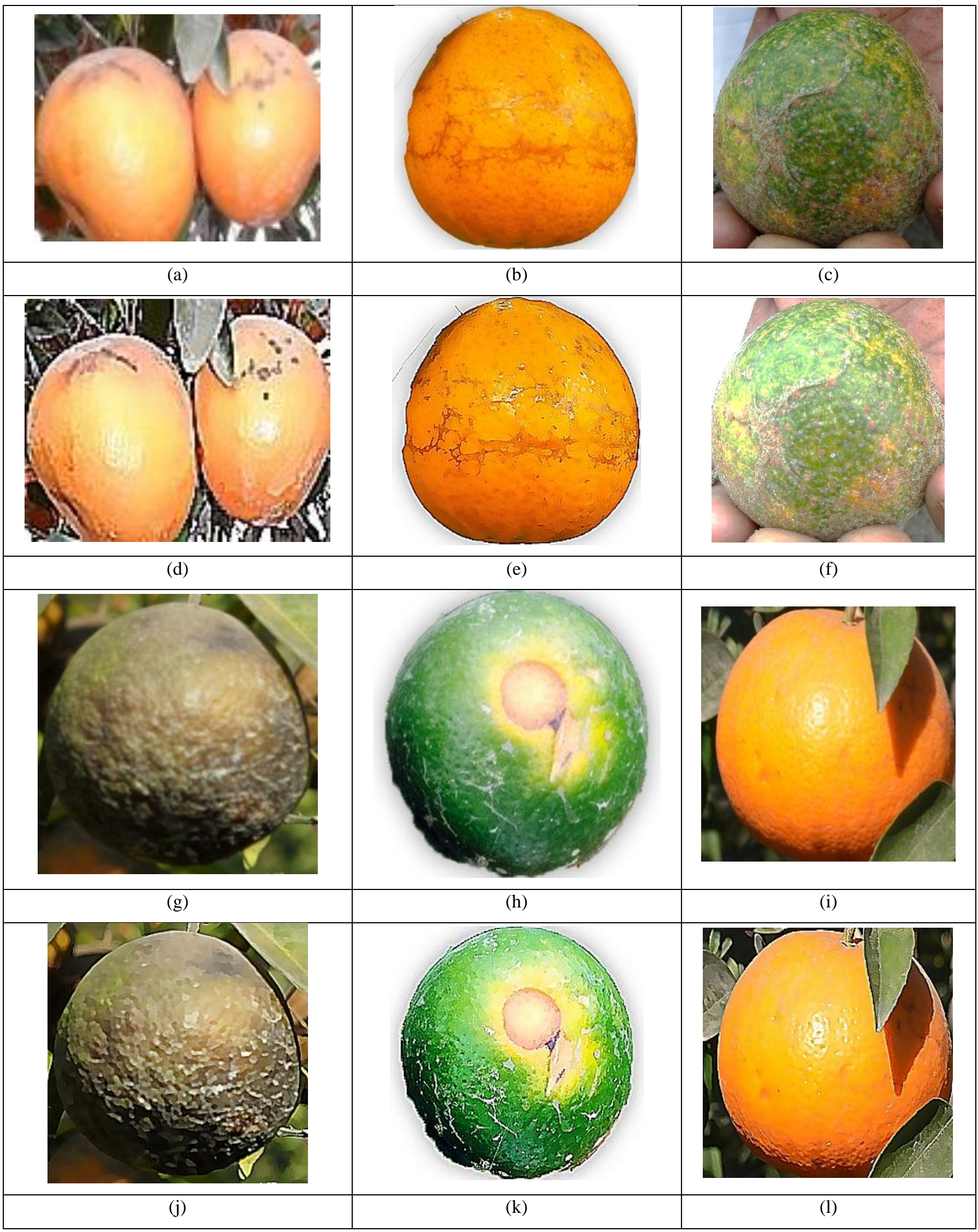

Published By:

Blue Eyes Intelligence Engineering \& Sciences Publication

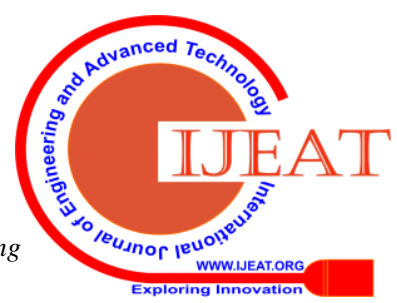


Table 3 displayed the images obtained from the Otsu based segmentation process. The images in Table (a-c) and (g-i) indicates the healthy images. The corresponding segmented images are found in the Table (d-f) and (j-l) respectively. The figures clearly indicate that the better segmentation images are found which will boost the classification outcome of the presented AGC-A model.

Table 3 Segmented Images

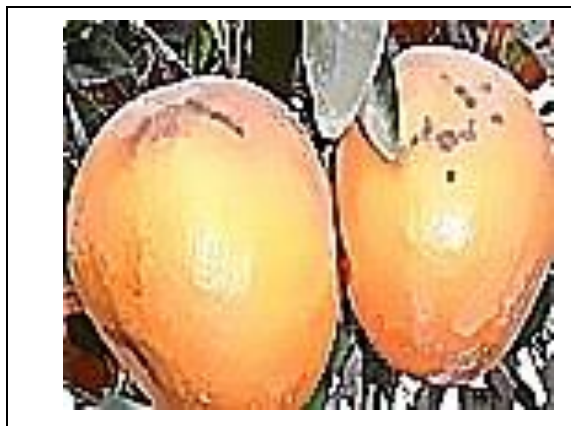

(a)

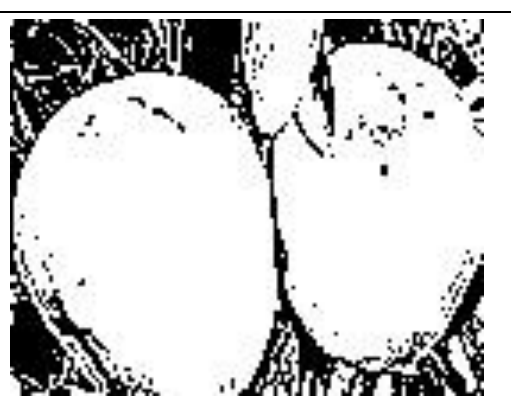

(d)

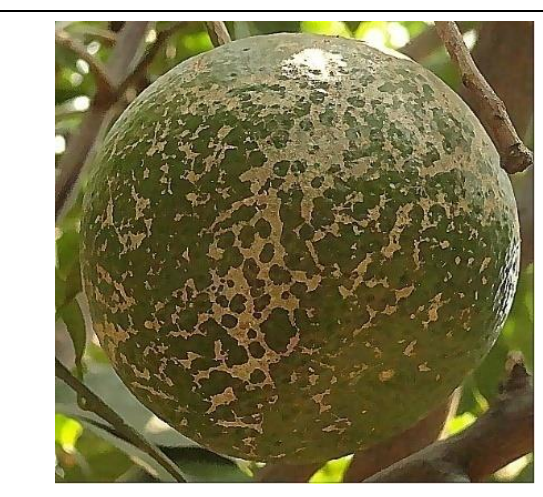

(g)

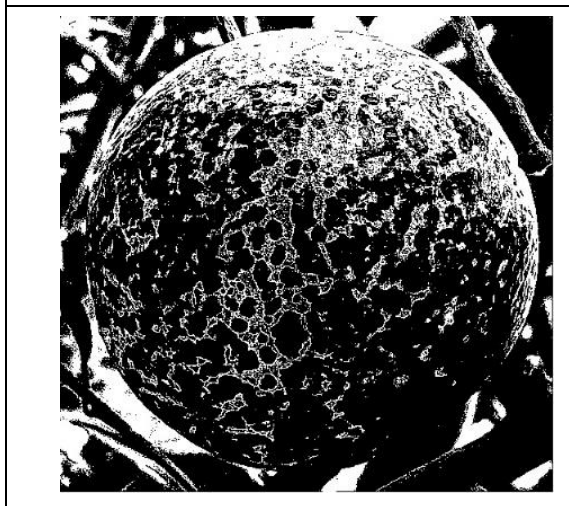

(j)

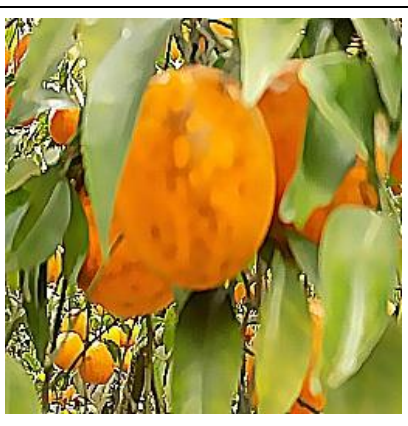

(b)

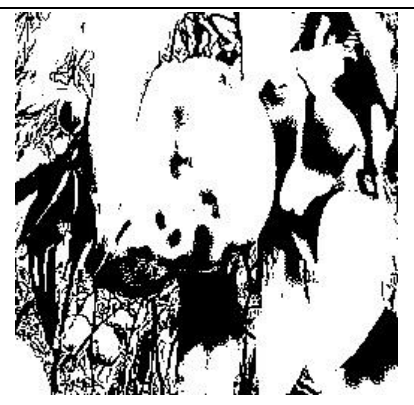

(e)

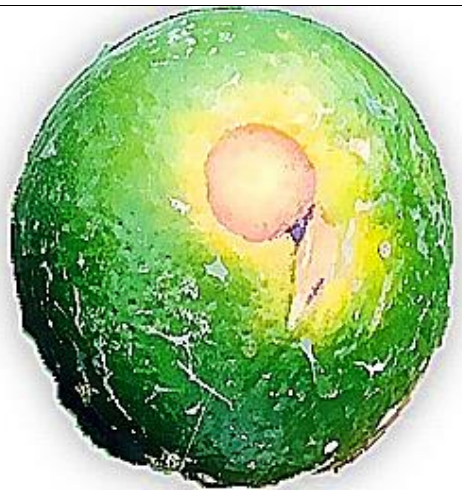

(h)

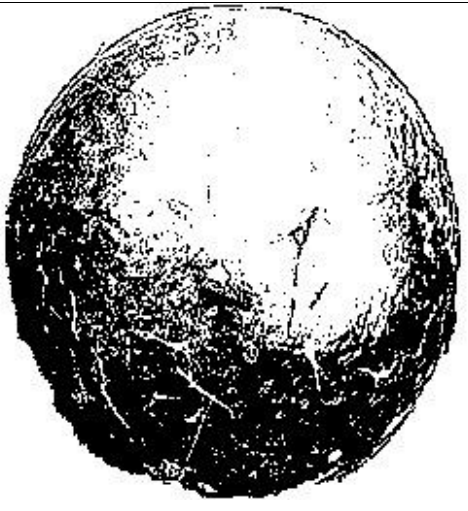

(k)

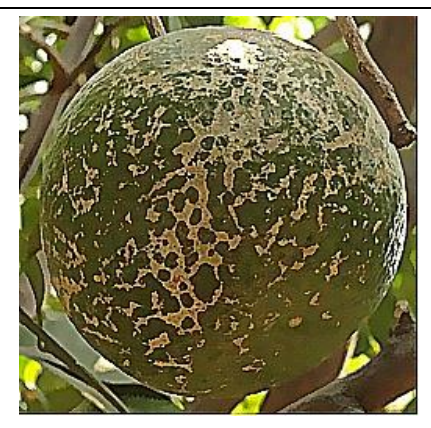

(c)

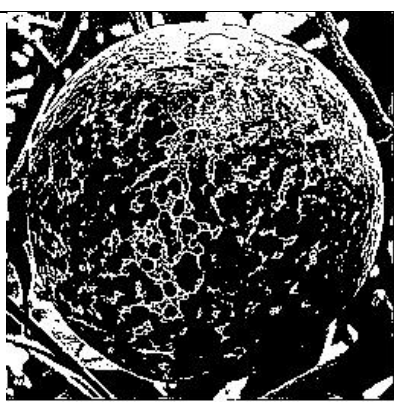

(f)

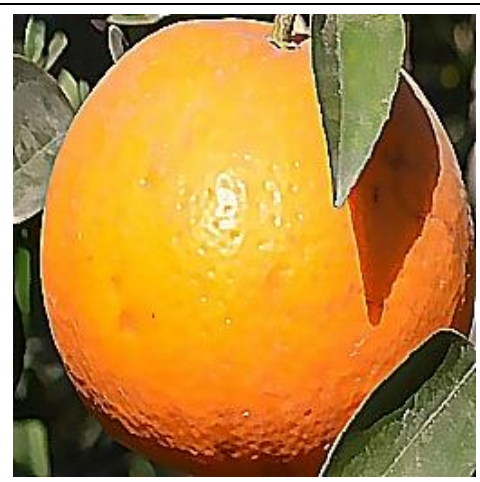

(i)

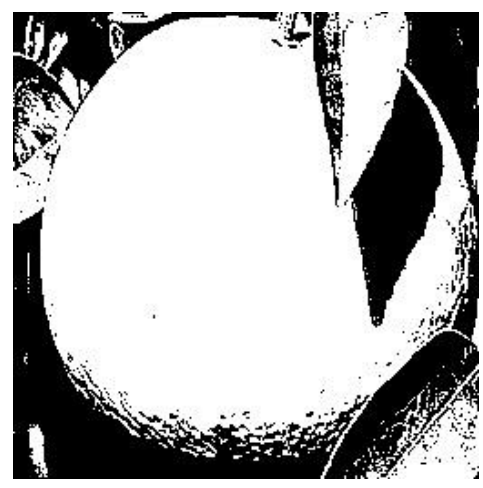

(l)

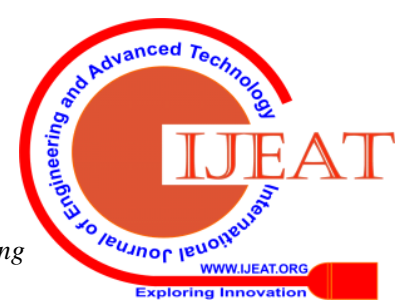




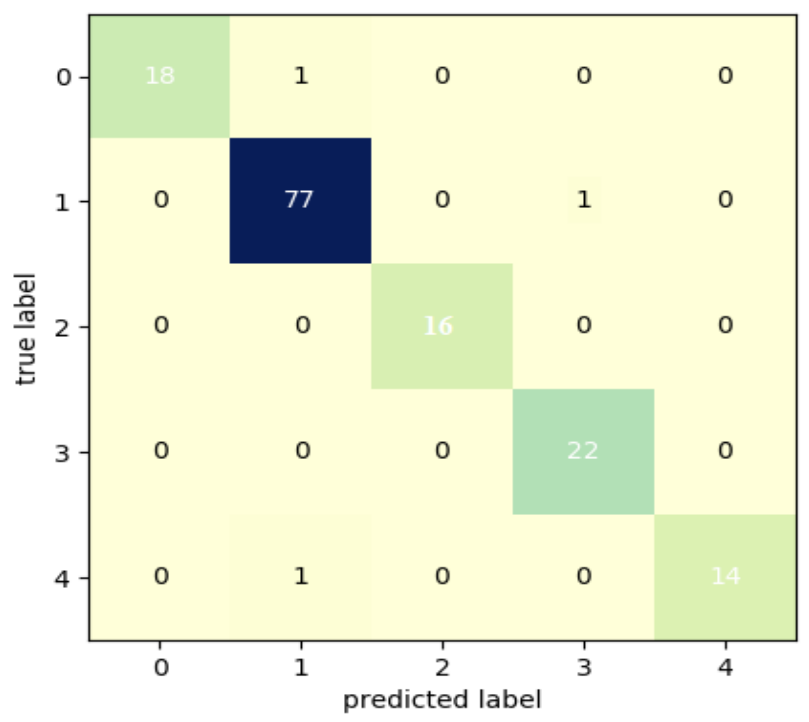

Fig. 4 shows the confusion matrix which is generated by the presented method. The confusion matrix in Fig. 4 is clearly represented in the form of $5 * 5$ matrix in Table 4 . The table value shows that the presented model clearly classifies a collection of 18 images under blackspot, 77 images under cancker, 16 images under greening, 22 images under healthy and 15 images under scab type. The values in Table 4 are manipulated in terms of TP, TN, FP and FN to determine the classification performance and is shown in Table 5 .

Fig.4. Generation of Confusion Matrix at Execution Time

Table 4 Confusion Matrix

\begin{tabular}{|l|l|l|l|l|l|l|}
\hline \multirow{2}{*}{ Input Label } & \multicolumn{7}{l}{ Different Classes of Citrus Fruits } & \multirow{2}{*}{ Total Images } \\
\cline { 2 - 7 } & Blackspot & Canker & Greening & Healthy & Scab & \\
\hline Blackspot & 18 & 1 & 0 & 0 & 0 & 19 \\
\hline Canker & 0 & 77 & 0 & 1 & 0 & 78 \\
\hline Greening & 0 & 0 & 16 & 0 & 0 & 16 \\
\hline Healthy & 0 & 0 & 0 & 22 & 0 & 22 \\
\hline Scab & 0 & 1 & 0 & 0 & 14 & 15 \\
\hline Total Images & 18 & 79 & 16 & 23 & 14 & 150 \\
\hline
\end{tabular}

Table 5 Manipulations from Confusion Matrix

\begin{tabular}{|c|c|c|c|c|c|}
\hline Different Classes & Blackspot & Canker & Greening & Healthy & Scab \\
\hline TP & 18 & 77 & 16 & 125 & 133 \\
\hline TN & 129 & 70 & 131 & 1 & 0 \\
\hline FP & 0 & 2 & 0 & 0 & 1 \\
\hline FN & 1 & 1 & 0 & & 0 \\
\hline
\end{tabular}

Table 6 Performance measures on different classes of citrus fruits for Proposed Method

\begin{tabular}{|l|l|l|l|l|l|l|l|l|}
\hline \multicolumn{1}{|c|}{ Classes } & FPR & Sensitivity & Specificity & Accuracy & F-score & FDR & G-Measure & Kappa \\
\hline Black Spot & 0 & 94.74 & 100 & 99.32 & 97.29 & 0 & 97.33 & 96.91 \\
\hline Canker & 2.77 & 98.71 & 97.22 & 98.00 & 98.08 & 2.53 & 98.09 & 95.99 \\
\hline Greening & 0 & 100 & 100 & 100 & 100 & 0 & 100 & 100 \\
\hline Healthy & 0.79 & 100 & 99.21 & 99.32 & 97.77 & 4.35 & 97.80 & 97.38 \\
\hline Scab & 0 & 93.33 & 100 & 99.32 & 96.55 & 0 & 96.61 & 96.18 \\
\hline
\end{tabular}


Table 6 and Fig. 5 provided the results attained by the presented AGC-A model on the identification of five different types of diseases. On the identification of black spot disease, the presented model clearly detects it with the minimum FPR and FDR of 0, maximum sensitivity of 94.74, specificity of 100, accuracy of 99.32, F-score of 97.29,
G-measure of 97.33 and kappa value of 96.91 respectively. On the identification of Canker disease, the presented model clearly detects it with the minimum FDR and FPR of 2.53 and 2.77, maximum Kappa of 95.99, specificity of 97.22, accuracy of 98.00, F-score of 98.08, G-measure of 98.09 and sensitivity value of 98.71 respectively.

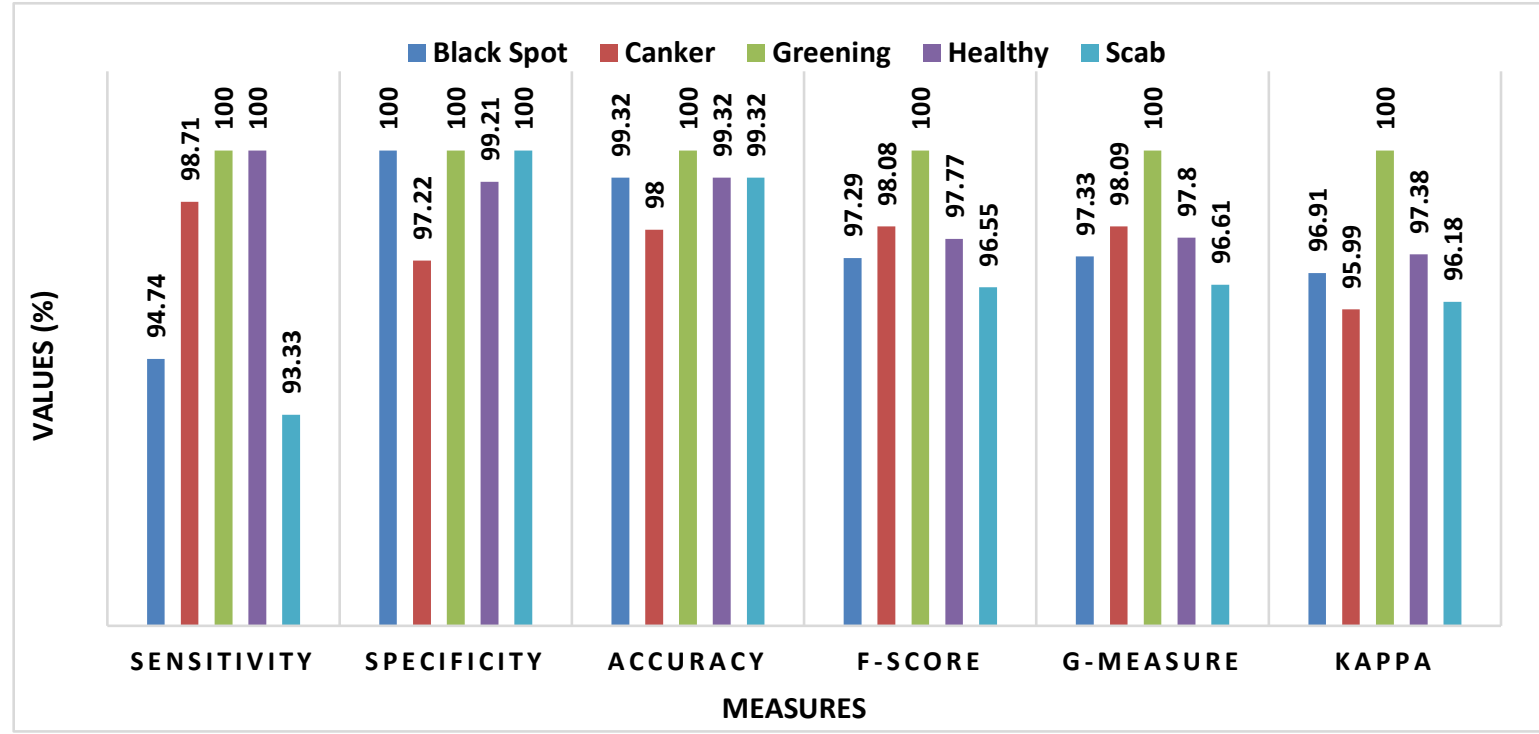

Fig. 5.Citrus disease Classification performance of proposed method

On the identification of Greening disease, the presented model clearly detects it with the minimum FPR and FDR of 0 , maximum sensitivity, specificity, accuracy, F-score, G-measure and kappa value of 100 respectively. On the identification of Healthy, the presented model clearly detects it with the minimum FPR and FDR of 0.79 and 4.35, maximum Kappa of 97.38, F-score of 97.77, G-Measure of 97.80, Specificity of 99.21, Accuracy of 99.32 and Sensitivity value of 100 respectively. On the identification of Scab disease, the presented model clearly detects it with the minimum FPR and FDR of 0, maximum sensitivity of 93.33, Kappa of 96.18, F-score of 96.55, G-Measure of 96.61, accuracy of 99.32 and Specificity value of 100 respectively.

Table 7 offers the results attained by various models on the detection of black spot diseases in terms of FPR, FDR and accuracy. On measuring the performance in terms of FPR and FDR, worse identification performance is exhibited by the LDA model with the maximum FPR and FDR values of 0.07 and 6.50 respectively. Simultaneously, the W-KNN model shows slightly better outcome with the FPR and FDR values of 0.06 and 6.40 respectively. Next to that, the EBT and DT models offers moderate as well as identical detection results with the same FPR and FDR values of 0.02 and 2.50 respectively. On continuing with, the M-SVM model offers competitive identification outcome with the minimum FPR and FDR values of 0.01 and 1.20 respectively. However, the presented AGC-A model offered optimal black spot identification with the least FPR and FDR value of 0 . With respect to accuracy, ineffective outcome is offered by LDA method with the minimum accuracy of 92.30 whereas the W-KNN method provided slightly higher accuracy value of 93.60. In the same way, the EBT and DT show near identical results with the accuracy values of 97.40 and 97.50 respectively. Besides, the M-SVM achieves somewhat higher accuracy value of 98.70 , the presented method shows supreme black spot disease identification with the highest accuracy value of 99.32 .

Table 7 Comparative results analysis for Black Spot identification

\begin{tabular}{|l|l|l|l|}
\hline Classes & FPR & FDR & Accuracy \\
\hline Proposed & 0 & 0 & 99.32 \\
\hline M-SVM [17] & 0.01 & 1.20 & 98.70 \\
\hline W-KNN & 0.06 & 6.40 & 93.60 \\
\hline EBT & 0.02 & 2.50 & 97.40 \\
\hline DT & 0.02 & 2.50 & 97.50 \\
\hline LDA & 0.07 & 6.50 & 92.30 \\
\hline
\end{tabular}

Table 8 offers the results attained by various models on the detection of canker diseases in terms of FPR, FDRand accuracy. On measuring the performance in terms of FPR and FDR, worse identification performance is exhibited by the EBT model with the maximum FPR and FDR values of 0.03 and 3.40 respectively. Simultaneously, the LDA model shows slightly better outcome with the FPR and FDR values of 0.02 and 2.60 respectively. However, the presented AGC-A model offered optimal canker identification with the least FPR and FDR value of 2.77 and 2.53. Next to that, the $\mathrm{W}-\mathrm{KNN}$ and DT models offers moderate as well as identical detection results with the same FPR and FDR values of 0.01 and 1.60 respectively. 
On continuing with, the M-SVM model offers competitive identification outcome with the minimum FPR and FDR values of 0.009 and 0.80 respectively. With respect to accuracy, ineffective outcome is offered by EBT method with the minimum accuracy of 96.50 whereas the LDA method provided slightly higher accuracy value of 97.40. The presented method shows supreme canker disease identification with the highest accuracy value of 98.00. In the same way, the W-KNN and DT show near identical results with the accuracy values of 98.30 respectively. Besides, the M-SVM achieves somewhat higher accuracy value of 99.10.

Table 8 Classification results of proposed with existing methods for Canker

\begin{tabular}{|l|l|l|l|}
\hline Classes & FPR & FDR & Accuracy \\
\hline Proposed & 2.77 & 2.53 & 98.00 \\
\hline M-SVM & 0.009 & 0.80 & 99.10 \\
\hline W-KNN & 0.01 & 1.60 & 98.30 \\
\hline EBT & 0.03 & 3.40 & 96.50 \\
\hline DT & 0.01 & 1.60 & 98.30 \\
\hline LDA & 0.02 & 2.60 & 97.40 \\
\hline
\end{tabular}

Table 9 offers the results attained by various models on the detection of scab diseases in terms of FPR, FDR and accuracy. On measuring the performance in terms of FPR and FDR, worse identification performance is exhibited by the EBT model with the maximum FPR and FDR values of 0.07 and 7.10 respectively. Simultaneously, the DT model shows slightly better outcome with the FPR and FDR values of 0.05 and 5.90 respectively. However, the W-KNN model offered optimal scab identification with the least FPR and FDR value of 0.04 and 4.50 respectively. Next to that, the LDA models offers moderate as well as detection results with the FPR and FDR values of 0.03 and 3.40 respectively. On continuing with, the M-SVM model offers competitive identification outcome with the minimum FPR and FDR values of 0.02 and 2.40 respectively. However, the presented AGC-A model offered optimal scab identification with the least FPR and FDR value of 0 . With respect to accuracy, ineffective outcome is offered by EBT method with the minimum accuracy of 92.90 whereas the DT method provided slightly higher accuracy value of 94.10. In the same way, the W-KNN show near results with the accuracy values of 95.30 respectively. Whereas, the LDA method provided slightly higher accuracy value of 96.50. Besides, the M-SVM achieves somewhat higher accuracy value of 97.60; the presented method shows supreme scab disease identification with the highest accuracy value of 99.32 .

Table 9 Classification results of proposed with existing methods for Scab

\begin{tabular}{|l|l|l|l|}
\hline Classes & FPR & FDR & Accuracy \\
\hline Proposed & 0 & 0 & 99.32 \\
\hline M-SVM & 0.02 & 2.40 & 97.60 \\
\hline W-KNN & 0.04 & 4.50 & 95.30 \\
\hline EBT & 0.07 & 7.10 & 92.90 \\
\hline DT & 0.05 & 5.90 & 94.10 \\
\hline LDA & 0.03 & 3.40 & 96.50 \\
\hline
\end{tabular}

Table 10 offers the results attained by various models on the detection of greening diseases in terms of FPR, FDR and accuracy. On measuring the performance in terms of FPR and FDR, worse identification performance is exhibited by the $\mathrm{W}-\mathrm{KNN}$ model with the maximum FPR and FDR values of 0.06 and 6.20 respectively.

Table 10 Classification results of proposed with existing methods for Greening.

\begin{tabular}{|l|l|l|l|}
\hline Classes & FPR & FDR & Accuracy \\
\hline Proposed & 0 & 0 & 100 \\
\hline M-SVM & 0.03 & 2.80 & 96.80 \\
\hline W-KNN & 0.06 & 6.20 & 93.80 \\
\hline EBT & 0.05 & 5.10 & 94.80 \\
\hline DT & 0.04 & 4.00 & 95.80 \\
\hline LDA & 0.05 & 5.10 & 94.80 \\
\hline
\end{tabular}

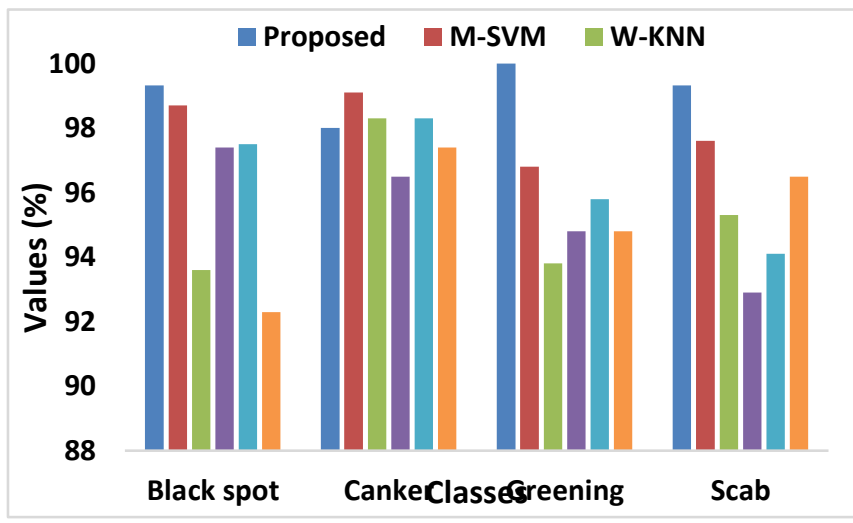

Fig.6. Comparative analysis of different Citrus disease identification models

Next to that, the EBT and LDA models offers moderate as well as identical detection results with the same FPR and FDR values of 0.05 and 5.10 respectively. Simultaneously, the DT model shows slightly better outcome with the FPR and FDR values of 0.04 and 4.00 respectively. On continuing with, the M-SVM model offers competitive identification outcome with the minimum FPR and FDR values of 0.03 and 2.80 respectively. However, the presented AGC-A model offered optimal greening identification with the least FPR and FDR value of 0 . With respect to accuracy, ineffective outcome is offered by W-KNN method with the minimum accuracy of 93.80. In the same way, the EBT and LDA show near identical results with the accuracy values of 94.80 whereas the DT method provided slightly higher accuracy value of 95.80 respectively. Besides, the M-SVM achieves somewhat higher accuracy value of 96.80 ; the presented method shows supreme greening disease identification with the highest accuracy value of 100 . Table 11 provided the outcome attained by different classifier models on the identification of all citrus diseases. Fig. 7 shows that the compared LDA model exhibits ineffective classification by attaining FPR, FNR and accuracy of 0.03, 6.75 and 93.50. 
Then, the slightly manageable results are attained by the presented W-KNN model with the FPR, FDR and accuracy values of $0.03,6.70$ and 93.80. In line with the EBT and DT offered moderate and identical outcome with the FR, FDR and accuracy values of 0.02, 5.65 and 94.50 .

Table 11 Classification results of proposed with existing methods

\begin{tabular}{|l|l|l|l|}
\hline Classes & FPR & FDR & Accuracy \\
\hline Proposed & 0.71 & 1.37 & 97.29 \\
\hline M-SVM & 0.02 & 4.68 & 95.80 \\
\hline W-KNN & 0.03 & 6.70 & 93.80 \\
\hline EBT & 0.02 & 5.65 & 94.50 \\
\hline DT & 0.02 & 6.08 & 94.50 \\
\hline LDA & 0.03 & 6.75 & 93.50 \\
\hline
\end{tabular}

At the same time, the M-SVM model offered near optimal results with the FPR, FDR and accuracy of 0.02, 4.68 and 95.80 respectively. Simultaneously, the presented model offered effective results with the FPR, FDR and accuracy of $0.71,1.37$ and 97.29 respectively.

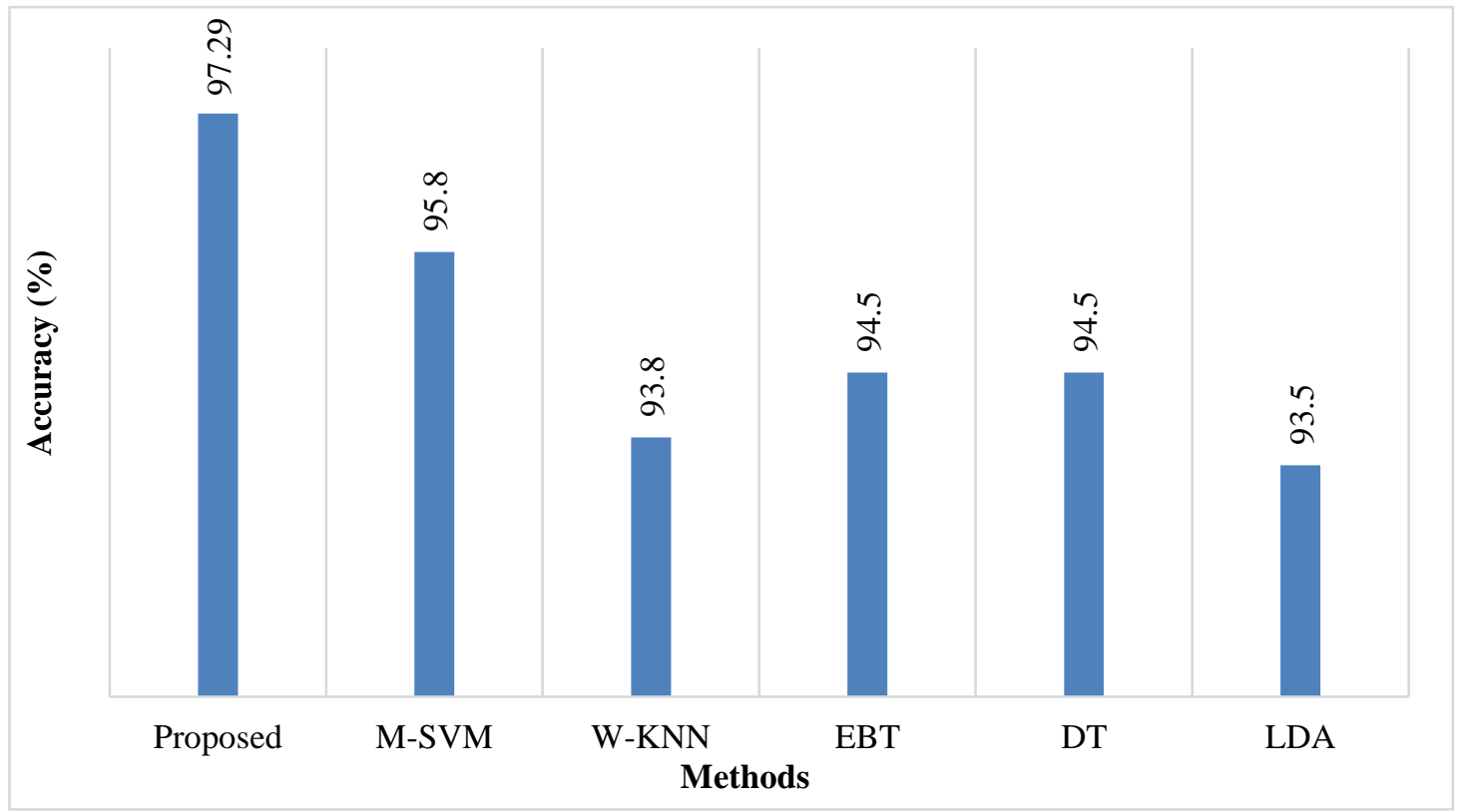

Fig.7. Comparative results analysis interms of accuracy

\section{CONCLUSION}

This paper has presented an effective DL based citrus disease detection and classification model by involving four main processes namely pre-processing, segmentation, feature extraction, and classification. Initially, pre-processing takes place to improve the quality of the image. Then, the Otsu method is applied to segment the images. Next, Alex-Net model is applied as a feature extractor. Finally, RF classifier is used to classify the different kinds of citrus diseases. For examining the performance of the applied AGA-A model, it is tested against Citrus Image Gallery dataset. The presented AGC-A model accurately classifies different types of citrus fruit diseases with the maximum detection rate of $97.29 \%$. In future, new deep learning model can be employed for classification task to further improve the detection performance.

\section{REFERENCES}

1. Malik, Z., et al., 2016. Detection and Counting of On-Tree Citrus Fruit for Crop Yield Estimation. IJACSA International Journal of Advanced Computer Science and Application. 7(5).

2. Gómez-Sanchis, J., et al., 2008. Automatic correction of the effects of the light source on spherical objects. An application to the analysis of hyperspectral images of citrus fruits. J. Food Eng. 85 (2), 191-200.

3. Omid, M., Khojastehnazhand, M., Tabatabaeefar, A., 2010. Estimating volume and mass of citrus fruits by image processing technique. J. Food Eng. 100 (2), 315-321.

4. Kumar, C., Chauhan, S., Alla, R.N., 2015. Classifications of citrus fruit using image processing-GLCM parameters. in Communications and Signal Processing (ICCSP), 2015 International Conference on IEEE.

5. Ali, H., et al., 2017. Symptom based automated detection of citrus diseases using color histogram and textural descriptors. Comput. Electron. Agric. 138, 92-104.

6. Wetterich, C.B., et al., 2016. Detection of citrus canker and Huanglongbing using fluorescence imaging spectroscopy and support vector machine technique. Appl. Optics 55 (2), 400-407.

7. Deng, X., et al., 2016. Citrus greening detection using visible spectrum imaging and CSVC. Comput. Electron. Agric. 130, 177-183. 
8. Stegmayer, G., et al., 2013. Automatic recognition of quarantine citrus diseases. Expert Syst. Appl. 40 (9), 3512-3517.

9. Zhang, M., Meng, Q., 2011. Automatic citrus canker detection from leaf images captured in field. Pattern Recogn. Lett. 32 (15), 2036-2046.

10. Gavhale, K.R., Gawande, U., Hajari, K.O., 2014. Unhealthy region of citrus leaf detection using image processing techniques. in Convergence of Technology (I2CT), 2014 International Conference for IEEE.

11. Cao, G., Huang, L., Tian, H., Huang, X., Wang, Y. and Zhi, R., 2018. Contrast enhancement of brightness-distorted images by improved adaptive gamma correction. Computers \& Electrical Engineering, 66, pp.569-582.

12. Zhang, B. and Allebach, J.P., 2008. Adaptive bilateral filter for sharpness enhancement and noise removal. IEEE transactions on Image Processing, 17(5), pp.664-678.

13. Zhang, J. and Hu, J., 2008, December. Image segmentation based on 2D Otsu method with histogram analysis. In 2008 International Conference on Computer Science and Software Engineering (Vol. 6, pp. 105-108). IEEE.

14. Krizhevsky, A., Sutskever, I. and Hinton, G.E., 2012. Imagenet classification with deep convolutional neural networks. In Advances in neural information processing systems (pp. 1097-1105).

15. Breiman, L., 2001. Random forests. Machine learning, 45(1), pp.5-32.

16. Citrus Diseases Image Gallery, December 20, 2017. [Online] Avaible http://idtools.org/id/citrus/diseases/gallery.php.

17. Sharif, M., Khan, M.A., Iqbal, Z., Azam, M.F., Lali, M.I.U. and Javed, M.Y., 2018. Detection and classification of citrus diseases in agriculture based on optimized weighted segmentation and feature selection. Computers and electronics in agriculture, 150, pp.220-234. 